\title{
Neuroanatomy of the vmPFC and dlPFC Predicts Individual Differences in Cognitive Regulation During Dietary Self-Control Across Regulation Strategies
}

\author{
Liane Schmidt, ${ }^{1}{ }^{\oplus}$ Anita Tusche, ${ }^{2}{ }^{-}$Nicolas Manoharan, ${ }^{3}{ }^{-}$Cendri Hutcherson, ${ }^{4,5}$ Todd Hare, ${ }^{6,7}$ \\ and $\odot$ Hilke Plassmann ${ }^{8,9}$ \\ ${ }^{1}$ Social and Affective Neuroscience team (SAN), Institute du Cerveau et de la Moelle Epinière, UMR 7225, U1127, Institut National de la Santé et de la \\ Recherche Médicale/Centre National de la Recherche Scientifique/Sorbonne Universités, Hôpital Pitié-Salpêtrière, 75013 Paris, France, ${ }^{2}$ Division of the \\ Humanities and Social Sciences, California Institute of Technology, Pasadena, California 91125, ${ }^{3}$ Sorbonne-Universités-INSEAD Behavioural Laboratory, \\ INSEAD, 75005 Paris, France, ${ }^{4}$ Department of Psychology, University of Toronto, Scarborough Campus, Toronto, Ontario M1C 1A4, Canada, ${ }^{5}$ Department \\ of Marketing, Rotman School of Management, University of Toronto, Toronto, Ontario M5S 3E6, Canada, ${ }^{6}$ Laboratory for Social and Neural Systems \\ Research, Department of Economics, University of Zurich, CH-8001 Zurich, Switzerland, ${ }^{7}$ Neuroscience Center Zurich, University of Zurich, Swiss Federal \\ Institute of Technology Zurich, 8092 Zurich, Switzerland, ${ }^{8}$ Marketing Area, INSEAD, 77305 Fontainebleau, France, and ${ }^{9}$ INSERM, U960 Laboratoire de \\ Neuroscience Cognitive, Ecole Normale Supérieure, 75005 Paris, France
}

Making healthy food choices is challenging for many people. Individuals differ greatly in their ability to follow health goals in the face of temptation, but it is unclear what underlies such differences. Using voxel-based morphometry, we investigated in healthy humans (i.e., men and women) the links between structural variation in gray matter volume and individuals' level of success in shifting toward healthier food choices. We combined MRI and choice data into a joint dataset by pooling across three independent studies that used a task prompting participants to explicitly focus on the healthiness of food items before making their food choices. Within this dataset, we found that individual differences in gray matter volume in the ventromedial prefrontal cortex (vmPFC) and dorsolateral prefrontal cortex (dlPFC) predicted regulatory success. We extended and confirmed these initial findings by predicting regulatory success out of sample and across tasks in a second dataset requiring participants to apply a different regulation strategy that entailed distancing from cravings for unhealthy, appetitive foods. Our findings suggest that neuroanatomical markers in the vmPFC and dlPFC generalized to different forms of dietary regulation strategies across participant groups. They provide novel evidence that structural differences in neuroanatomy of two key regions for valuation and its control, the vmPFC and dIPFC, predict an individual's ability to exert control in dietary choices.

Key words: cognitive regulation success; dietary self-control; dlPFC; valuation; vmPFC; voxel-based morphometry

\section{Significance Statement}

Dieting involves regulating food choices to eat healthier foods and fewer unhealthy foods. People differ dramatically in their ability to achieve or maintain this regulation, but it is unclear why. Here, we show that individuals with more gray matter volume in the dorsolateral and ventromedial prefrontal cortex are better at exercising dietary self-control. This relationship was observed across four different studies examining two different forms of dietary self-regulation, suggesting that neuroanatomical differences in the ventromedial prefrontal cortex and dorsolateral prefrontal cortex may represent a general marker for self-control abilities. These results identify candidate neuroanatomical markers for dieting success and failure, and suggest potential targets for therapies aimed at preventing or treating obesity and related eating disorders.

\section{Introduction}

Humans have a remarkable capacity to use various cognitive regulation strategies to attain desired goals and to exercise selfcontrol (Kober et al., 2010). Self-control dilemmas are often characterized by a trade-off between an immediate, tempting reward and a delayed, more abstract one (McClure et al., 2004; Kable and Glimcher, 2007; Hare et al., 2009, 2011; Li et al., 2013; e.g., eat a piece of tasty chocolate cake now or forgo the pleasure 
Table 1. Study and dataset overview

\begin{tabular}{|c|c|c|c|c|c|c|c|c|c|c|}
\hline Study & Dataset & Local ethics committee & Scanner & MPRAGE sequence & $N$ & Age, years (SEM) & Female/male, $n$ & Task condition & DV & Other ratings \\
\hline 1 & 1 & $\begin{array}{l}\text { California Institute of } \\
\text { Technology (Pasadena, CA) }\end{array}$ & $\begin{array}{l}3 \text { T Trio } \\
\text { Siemens }\end{array}$ & $\begin{array}{c}\mathrm{TR}=1.5 \mathrm{~s} ; \mathrm{TE}=3.05 \mathrm{~ms} ; \\
176 \text { sagittal slices; } \\
256 \times 256 \text { matrix }\end{array}$ & $13^{*}$ & $38.2(12.8)$ & $8 / 5$ & $\begin{array}{l}\text { Health, natural, } \\
\text { taste }\end{array}$ & SV & Health, taste \\
\hline 2 & 1 & $\begin{array}{c}\text { California Institute } \\
\text { of Technology }\end{array}$ & $\begin{array}{l}3 \text { T Trio } \\
\text { Siemens }\end{array}$ & $\begin{array}{c}\mathrm{TR}=1.5 \mathrm{~s} ; \mathrm{TE}=2.91 \mathrm{~ms} ; \\
176 \text { sagittal slices; } \\
256 \times 256 \text { matrix }\end{array}$ & 35 & $29(0.9)$ & $16 / 19$ & $\begin{array}{l}\text { Health, natural, } \\
\text { taste }\end{array}$ & SV & Health, taste \\
\hline 3 & 1 & $\begin{array}{l}\text { Comité de Protection des } \\
\text { Personnes, lle-de-France VI, } \\
\text { INSERM approval \#C07-28, } \\
\text { DGS approval \#2007-0569, } \\
\text { ID-RCB approval \#2007- } \\
\text { A01125-48CPP }\end{array}$ & $\begin{array}{l}3 \text { T Verio } \\
\text { Siemens }\end{array}$ & $\begin{array}{c}\mathrm{TR}=2.3 \mathrm{~s} ; \mathrm{TE}=2.98 \mathrm{~ms} ; \\
176 \text { sagittal slices; } \\
240 \times 256 \text { matrix }\end{array}$ & 43 & $24.8(5.1)$ & 43 & $\begin{array}{l}\text { Health, natural, } \\
\text { taste }\end{array}$ & SV & Health, taste \\
\hline 4 & 2 & $\begin{array}{l}\text { California Institute } \\
\text { of Technology }\end{array}$ & $\begin{array}{l}3 \text { T Trio } \\
\text { Siemens }\end{array}$ & $\begin{array}{l}\text { TR }=1.5 \mathrm{~s} ; \\
\text { TE = } 3.05 \mathrm{~ms} ; \\
176 \text { sagittal slices; } \\
256 \times 256 \text { matrix }\end{array}$ & 32 & $22(3.3)$ & $11 / 21$ & $\begin{array}{l}\text { Distance, natural, } \\
\text { indulge }\end{array}$ & WTP & Food liking \\
\hline
\end{tabular}

DV, Dependent variable; ID-RCB, Registration number of the study; DGS, Direction Générale de la Santé.

*Note that information on the gender and age for 20 of the original 33 participants in the study by Hare et al. (2011) was no longer available. Therefore, we included only the 13 participants from that study for whom we had all relevant information for the data analysis.

to achieve better health and a longer life in the future). Such decisions about diet, exercise, and other reward-guided behaviors all have consequential long-term effects on health and wellbeing. However, many people struggle to consistently stick to their diets, exercise, and save for retirement. A key challenge for promoting healthy, adaptive decision-making is understanding what underlies individual differences in self-control success (Tangney et al., 2004; Saarni et al., 2006; Pietiläinen et al., 2012; Holmes et al., 2016).

Recent work in cognitive neuroscience has investigated this question by examining how individual differences in functional brain activity during regulation tasks can be linked to differences in self-control abilities. For example, trait measures of selfcontrol correlated with both the ability to regulate negative emotions and enhanced functional connectivity between the amygdala and dorsolateral prefrontal cortex (dlPFC; Paschke et al., 2016). Other studies have linked the desire for immediate reward to attenuated functional connectivity between cognitive control and reward-related brain regions such as the anterior prefrontal cortex and nucleus accumbens (Diekhof and Gruber, 2010; Diekhof et al., 2012; van den Bos et al., 2014; MorenoLopez et al., 2016). These findings are in line with work associating self-control abilities with connectivity of resting-state brain networks. For example, self-control when making trade-offs between smaller, sooner monetary rewards and larger, later ones was linked to enhanced resting-state connectivity between neural pathways underpinning reward processing and cognitive regulation processes ( $\mathrm{Li}$ et al., 2013).

Although associations between functional activation and self-control are tantalizing, it is unclear whether individual differences in success are driven by momentary fluctuations in motivation or attention, or by more stable, potentially neuroanatomical, differences in the mechanisms of choice. Initial support for a neuroanatomical basis comes from studies linking individual differences in structural connectivity between reward-related and cognitive control areas to behavioral differences in impa-

The authors declare no competing financial interests.

Correspondence should be addressed to Liane Schmidt, Institute du Cerveau et de la Moelle Epinière, Hôpital Pitié-Salpêtrière, 47 Boulevard de I'Hôpital, 75013 Paris, France. E-mail: liane.schmidt@icm-institute.org.

DOI:10.1523/JNEUROSCI.3402-17.2018

Copyright $\odot 2018$ the authors $\quad 0270-6474 / 18 / 385800-08 \$ 15.00 / 0$ tience for receiving monetary rewards (Peper et al., 2013; van den Bos et al., 2014). The goal of the current study was to further test this idea by investigating (1) whether differences in neuroanatomy predict an individual's ability to regulate healthier dietary choices, and, if so, (2) whether such differences depend on the type of regulatory strategy or are generalizable across different strategies promoting healthier choices and participant populations.

To answer these questions, we used voxel-based morphometry (VBM) to determine whether and where neuroanatomical differences predict regulatory success during dietary decisions that involve explicitly focusing on health goals. First, we aggregated data from three independent studies (i.e., dataset 1), all using a similar task that prompted participants to regulate their dietary decision processes by focusing on the healthiness of foods. Because subjective experience and behavior can be modified by using distinct strategies with distinct consequences (Gross, 1998), we then tested whether the same neuroanatomical variation underlies regulatory success for a different regulation strategy. We addressed this second question by examining structural predictors of regulatory success in a fully independent fourth study (i.e., dataset 2): participants in this study were not told to focus specifically on health attributes, but were instead encouraged to use a self-selected strategy to distance themselves from and reduce cravings for tasty but unhealthy foods (Hutcherson et al., 2012).

Our results indicate that neuroanatomical differences in specific value-related and cognitive control areas in the ventromedial prefrontal cortex (vmPFC) and the dlPFC are generally predictive of regulatory success across different strategies and independent populations. They thus hold promise to serve as neuroanatomical markers of the ability to exercise self-control over dietary decisions.

\section{Materials and Methods}

Participants. The analyses included 123 healthy individuals (mean age, $29.97 \pm 0.96$ years; 78 females, 45 males) from three different previously published studies (Hare et al., 2011; Hutcherson et al., 2012; Tusche and Hutcherson 2018) and one different unpublished studies. Research was conducted in accordance with the Helsinki Declaration and was approved by the local ethics committee (for an overview, see Table 1). All participants provided written and informed consent. Participants were screened for the following standard fMRI inclusion criteria: right- 
a

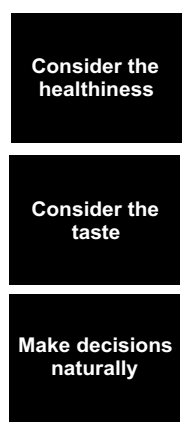

$5 \mathrm{~s}$
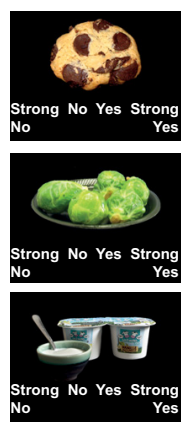

RT
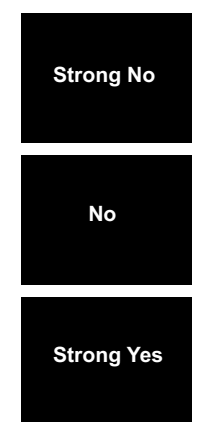

$0.5 \mathrm{~s}$
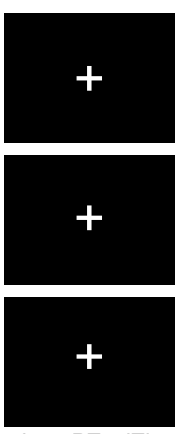

$3 s-R T+I T I$

C

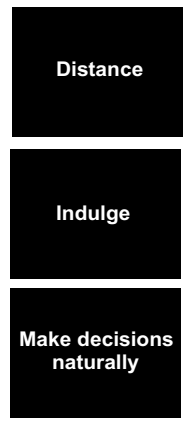

$2 \mathrm{~s}$
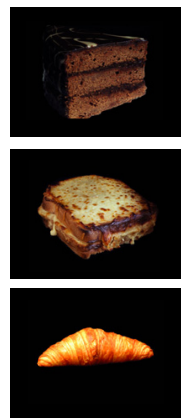

$4 \mathrm{~s}$
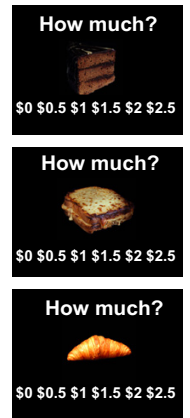

$2 \mathrm{~s}$ b

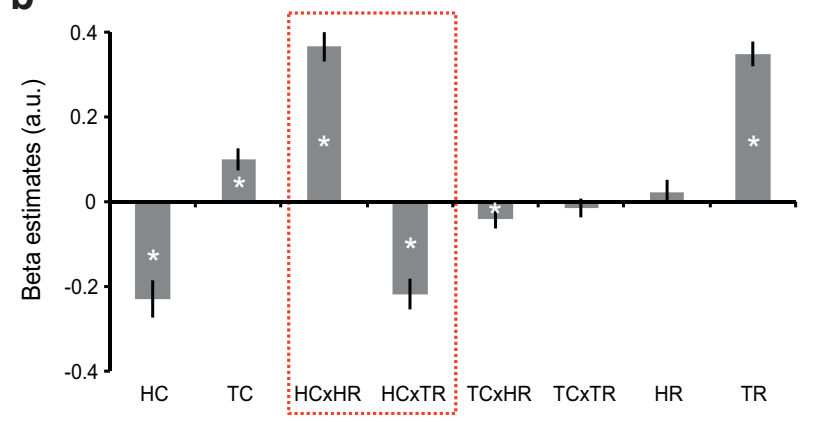

d

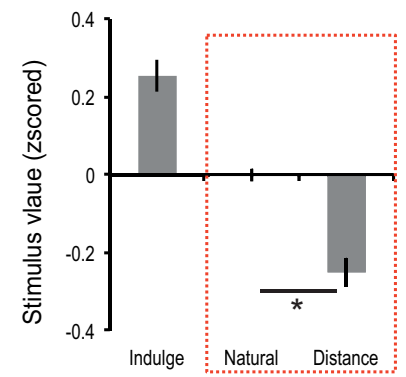

Figure 1. Experimental design and behavioral results. $\boldsymbol{a}$, Behavioral task dataset 1. Screenshots display successive events within one trial of each condition [i.e., health focus (i.e., HC), taste focus (i.e., TC), and natural focus (i.e., NC) conditions] during the dietary decision-making task performed by the participants of dataset 1 with durations in seconds. Conditions were presented in blocks, randomly intermixed. Each block started with an instruction to focus attention on the healthiness, taste, or natural preference. Next, a food item was displayed on the screen and participants had to evaluate how much they would like to eat it by pressing buttons corresponding to "strong no," "no," "yes," and "strong yes." $\boldsymbol{b}$, Behavioral results in dataset 1 ( $N=91$ ). The bar graph depicts mean $\beta$ estimates for each regressor of Equation 1. The dotted red lines indicate the behavioral measures of interest: the weight of the healthiness (i.e., HR) and the tastiness (i.e., TR) on stimulus value computation during the HC. c, Behavioral task dataset 2. Screenshots display successive events within one trial of each condition (i.e., DC, IC, and NC conditions) during the dietary decision-making task performed by the participants of dataset 2 with durations in seconds. Conditions were presented in blocks, randomly intermixed. Each block started with an instruction to try to distance oneself from food cravings, indulge in food cravings, or make decisions naturally. Next, a food item was displayed on the screen and participants had to evaluate how much they would be willing to pay for the food item by pressing buttons corresponding to $\$ 0, \$ 0.50, \$ 1, \$ 1.50, \$ 2$, and $\$ 2.50$. $d$, Behavioral results in dataset $2(N=32)$. The bar graph depicts the mean stimulus value of food items in each condition. The asterisks $(*)$ indicate significance against zero at $p<0.05$. Error bars indicate \pm intersubject SEM.

handedness, normal to corrected-to-normal vision, no history of substance abuse or any neurological or psychiatric disorder, and no medication or metallic devices. All participants were tested after $4 \mathrm{~h}$ of fasting.

Procedure. Participants took part in one of two different dietary decision-making tasks that required them to use various strategies to make healthier choices.

Regulation task 1: focusing on healthiness of foods (dataset 1). Dataset 1 included 91 participants pooled over three similar studies [study 1: $N=$ 13 (Hare et al., 2011); study 2: $N=35$ (Tusche and Hutcherson, 2018); study 3: $N=43$ (Schmidt et al., 2015); Table 1]. Participants decided while in the fMRI scanner how much they would like to eat different food items varying in tastiness and healthiness at the end of the experiment. Participants made their choices under the following three different conditions: being prompted to focus on the (1) tastiness condition (TC) or (2) healthiness condition (HC) of the foods, or (3) with no dieting instruction [NC; i.e., making food choices as they naturally would, which served as a baseline; Fig. 1a]. Participants always started with a baseline block (NC) followed by a randomized taste or health block. The conditions were randomized across blocks of 10 trials, and participants were instructed to rate how much they wanted to eat a food item presented on the screen relative to a constant default option chosen for each participant. To determine the weight participants placed on the tastiness and healthiness of a food under different regulatory goals, participants also indicated the perceived healthiness and tastiness of all presented foods using a 4 point Likert scale (outside the scanner).

The tasks in studies 1, 2, and 3 were identical, with two exceptions. First, studies 1 and 3 consisted of 18 blocks of 10 trials (i.e., 6 blocks per condition of HC, TC, and NC), for a total of 180 trials. Study 2 consisted of 27 blocks of 10 trials (i.e., nine blocks per condition of HC, TC, and $\mathrm{NC}$ ), for a total of 270 trials. Moreover, in study 2 the same food pictures were presented once in each condition of HC, TC, and NC. Second, studies 1 and 2 included both men and women. Study 3 included only female participants, who served as lean control subjects in a large-scale project aiming at the neural and behavioral underpinnings of dietary decision-making in female obesity.

Regulation task 2: distancing oneself from cravings for unhealthy foods (dataset 2). In a fourth study, 32 participants completed a different dietary self-control task (Hutcherson et al., 2012). In study 4, rather than explicitly considering the healthiness of food items, participants were instructed to distance themselves [distance condition (DC)] from food cravings when contemplating highly palatable foods rich in calories (Fig. 1c). (In separate blocks, participants in this study also attempted to indulge their cravings for palatable, unhealthy foods; given the focus of this article on healthy food choices, these trials were not included in the current analyses.) Participants were told to regulate their cravings by applying any strategy they preferred. The task also had a baseline condi- 
tion in which participants were asked to make their dietary decisions naturally, without any regulation instruction (i.e., NC). Fifty trials of each of the three conditions were randomly intermixed, for a total of 150 trials. To make their decisions, participants were asked to use a 6 point scale ( $\$ 0, \$ 0.50, \$ 1, \$ 1.50, \$ 2, \$ 2.50)$ to indicate their willingness to pay (WTP) for the right to eat the food at the end of the experiment, rather than being asked about how much they would like to eat it. Importantly, participants rated all foods for subjective liking before entering the scanner, on the same scale used for dataset 1 . The high correlation between prescan liking and in-scan bids for foods in the natural condition (average $r=0.72 \pm 0.19 ; p<0.001)$ suggested that they measured similar constructs.

To incentivize participants to choose according to their actual preferences, in all four studies participants had to eat one item at the end of the experiment that was determined by a random draw of one trial. Food pictures were presented on a computer screen in the form of highresolution pictures (72 dpi). Matlab and Psychophysics Toolbox extensions were used for stimulus presentation and response recording. Participants saw the stimuli via goggles or a head coil-based mirror and indicated their responses using a response box system.

Behavioral analyses. All statistical tests were conducted with the Matlab Statistical Toolbox (Matlab 2014a, MathWorks). In dataset 1, we measured regulatory success by combining the increase in weight given to healthiness and the decrease in weight given to tastiness during the health focus condition (i.e., HC), following the approach of Hare et al., 2011. To this end, we fit a general linear model (GLM) to stimulus value (SV; i.e., participants' ratings of how much they would like to eat a food item). The behavioral GLM is described by Equation 1, as follows:

$$
\begin{aligned}
\mathrm{SV}= & \beta_{0}+\beta_{H C} \mathrm{HC}+\beta_{T C} \mathrm{TC}+\beta_{H R} \mathrm{HR}+\beta_{T R} \mathrm{TR}+\beta_{H C \times H R} \mathrm{HC} \times \mathrm{HR} \\
& +\beta_{H C \times T R} \mathrm{HC} \times \mathrm{TR}+\beta_{T C \times H R} \mathrm{TC} \times \mathrm{HR}+\beta_{T C \times T R} \mathrm{TC} \times \mathrm{TR}+\epsilon
\end{aligned}
$$

SV corresponded to the dependent variable, which was predicted by the following regressors: $\mathrm{HC}$, an indicator variable for a health focus condition block (dummy coded); TC, an indicator variable for the taste focus condition block (dummy coded); and health rating (HR) and taste rating (TR) for the trial-specific food item (assessed outside the scanner). This GLM also included the following four interaction terms: health focus condition by health rating $(\mathrm{HC} \times \mathrm{HR})$; health focus condition by taste rating $(\mathrm{HC} \times \mathrm{TR})$; taste focus condition by health rating $(\mathrm{TC} \times \mathrm{HR})$; and taste focus condition by taste rating (TC $\times$ TR). Note that the TR and HR regressors measure to what extent taste and health attributes of the food stimuli influenced participants' stimulus values during the baseline NC. SV, TR, and HR regressors were scaled as -2 (strong no), -1 (no), 1 (yes), or 2 (strong yes). In contrast, the interaction terms $(\mathrm{HC} \times \mathrm{HR}$, $\mathrm{HC} \times \mathrm{TR}, \mathrm{TC} \times \mathrm{HR}$, and TC $\times \mathrm{TR}$ ) assessed how much change occurred in the weight given to the taste and health attributes during the health or taste focus conditions, respectively. The individual regression coefficients (i.e., $\beta$ estimates) for each regressor were analyzed at the group level using one-sample, two-tailed $t$ tests.

For the purpose of our subsequent analyses, Equation 1 contains two terms of interest that characterize how participants regulated their food decisions to make healthier choices in the HC, as follows: (1) HC $\times \mathrm{HR}$, which assessed how much more participants integrated the healthiness of the food; and (2) $\mathrm{HC} \times \mathrm{TR}$, which assessed how much the tastiness of the food was inhibited during the food decision. Because these two measures were highly correlated $(r=0.53, p<0.001)$, we integrated them into an overall regulatory success score that was then entered as a regressor in the VBM analysis (i.e., Regulatory Success dataset $1=\beta_{\mathrm{HC} \times \mathrm{HR}}-\beta_{\mathrm{HC} \times \mathrm{TR}}$ ). The more positive this difference score is, the higher the regulatory success of the participant.

The difference in SV (measured in this task as participants' WTP) between the natural condition and the distance condition was used as the measure of regulatory success (Regulatory Success dataset2 $=\mathrm{SV}_{\mathrm{NC}}-$ $\mathrm{SV}_{\mathrm{DC}}$ ) for the 32 participants who took part in the second dietary decision-making task (i.e., dataset 2). This approach is the same as that originally used by Hutcherson et al. (2012). A positive score indicated that participants successfully regulated their cravings and exercised selfcontrol because their SV for unhealthy foods was lower when they distanced themselves from their food cravings compared with their natural responses. A paired, two-tailed $t$ test was conducted to test for a significant difference in SV between the distance and natural conditions.

MRI structural acquisition. Anatomical brain images were collected on a $3 \mathrm{~T}$ Trio Siemens (studies 1, 2, and 4) or a $3 \mathrm{~T}$ Verio Siemens scanner (study 3). Whole-brain high-resolution T1-weighted structural scans $(1 \times 1 \times 1 \mathrm{~mm})$ were acquired for all 123 participants with an MPRAGE sequence. Details of the sequences are described in Table 1.

MRI data preprocessing. Each participant's anatomical image was segmented into gray matter (GM) using the SPM12 [Wellcome Trust Center for Neuroimaging, University College London, London, UK (http:// www.fil.ion.ucl.ac.uk/spm)] segmentation tool. Individual GM images were then coregistered between participants using Diffeomorphic Anatomical Registration through Exponentiated Lie Algebra (DARTEL). Next, the registered images were normalized to the Montreal Neurological Institute (MNI) stereotactic space using the DARTEL template, and spatially smoothed using a Gaussian kernel with full-width at halfmaximum of $8 \mathrm{~mm}$.

VBM analyses. All VBM analyses were performed using SPM12. Outof-sample predictions were conducted using the glmfit and glmval functions from the Matlab Statistical Toolbox (Matlab 2014a, MathWorks). We conducted GLM-based leave-one-subject-out (LOSO) predictive analyses within dataset 1 as well as cross-study predictions between datasets 1 and 2 to test whether individual differences in neuroanatomy were linked to dietary self-control choices. Building on the fMRI literature, our a priori focus was on GM volume in the dIPFC and vmPFC, but we also tested models including additional regions for completeness. The details of the various analysis steps are given in the following paragraphs.

GM volume-based predictions of regulatory success within dataset 1 . We conducted an out-of-sample LOSO prediction analysis for all participants in dataset 1 using the GLM described in Equation 2, as follows:

$$
\begin{aligned}
\text { GMVolume }=\beta_{0}+ & \beta_{\text {reg_success }}+\beta_{\text {age }}+\beta_{\text {gender }}+\beta_{\text {scanner }} \\
& +\beta_{\text {study } 1}+\beta_{\text {study } 2}+\beta_{\text {study } 3}+\beta_{\text {globalGM }}+\epsilon
\end{aligned}
$$

The $\beta$ estimate ( $\beta_{\text {reg_success }}$ ) quantifying the relationship between the change in regulatory success during the health focus condition [i.e., $\left(\beta_{\mathrm{HC} \times \mathrm{HR}}-\beta_{\mathrm{HC} \times \mathrm{TR}}\right)$ from the behavioral regression (Eq. 1)] and voxelwise GM volume was our effect of interest. Note that regulatory success is expected to increase with a positive value for $\beta_{\mathrm{HC} \times \mathrm{HR}}$ or a negative value for $\beta_{\mathrm{HC} \times \mathrm{TR}}$, so the subtraction $\left(\beta_{\mathrm{HC} \times \mathrm{HR}}, \beta_{\mathrm{HC} \times \mathrm{TR}}\right)$ quantifies the total increase in regulatory success. Voxels in which GM volume was potentially predictive of regulatory success were identified by the contrast $\left[\beta_{\text {reg_success }}>0\right]$. To control for variance related to age, gender, MRI scanner, study, and global GM volume, these factors were included in all voxelwise linear regression models (following ANCOVA normalization).

The LOSO procedure was conducted as follows: we divided dataset 1 into 91 separate training sets (90 participants) and test sets ( 1 participant). For each training set, we computed the GLM described by Equation 2 above. We then created 91 sets of regions of interest (ROIs) from these results using a voxelwise threshold of $t=2.64(p<0.005)$. Each set of contiguous voxels was treated as a single ROI, and GM volume was averaged over the voxels in each ROI. Next, we used these 91 sets of independently defined ROI masks to calculate a predicted regulatory success measure for each participant in dataset 1 using the GLMs in Equations 3 and $3_{\text {all }}$. These GLMs differed in terms of whether they used only our a priori ROIs, dIPFC and vmPFC, or all ROIs identified in a particular training set to predict regulatory success in the left-out participant, as follows:

regulatory success $=\beta_{0}+\beta_{d l P F C} G_{d l P F C}+\beta_{v m P F C} * G M_{v m P F C}$

regulatory success $=\beta_{0}+\beta_{d l P F C} * G M_{d l P F C}+\beta_{v m P F C} * G M_{v m P F C}$

$$
+\beta x * G M x+\epsilon \quad\left(3_{\text {all }}\right)
$$


Table 2. Multiple regression results on SV in dataset 1

\begin{tabular}{|c|c|c|c|c|c|c|c|c|c|}
\hline & Intercept & $H R$ & TR & $\mathrm{HC}$ & $\mathrm{TC}$ & $\mathrm{HC} \times \mathrm{HR}$ & $\mathrm{TC} \times \mathrm{HR}$ & $\mathrm{HC} \times \mathrm{TR}$ & $\mathrm{TC} \times \mathrm{TR}$ \\
\hline \multicolumn{10}{|l|}{ Study 1} \\
\hline Coeff & -0.01 & 0.14 & 0.61 & -0.20 & -0.01 & 0.24 & -0.06 & -0.20 & 0.05 \\
\hline STE & 0.07 & 0.04 & 0.05 & 0.06 & 0.04 & 0.05 & 0.03 & 0.06 & 0.03 \\
\hline$t$ & -0.12 & 3.88 & 13.25 & -3.36 & -0.17 & 4.93 & -2.02 & -3.67 & 1.59 \\
\hline$Z$ & -1.32 & 3.27 & 7.50 & -2.86 & -1.11 & 4.02 & -1.61 & -3.12 & 1.16 \\
\hline$p$ & 0.9061 & 0.0005 & 0.0000 & 0.0021 & 0.8656 & 0.0000 & 0.0532 & 0.0009 & 0.1231 \\
\hline \multicolumn{10}{|l|}{ Study 2} \\
\hline Coeff & 0.24 & -0.06 & 0.26 & -0.28 & 0.08 & 0.28 & -0.06 & -0.19 & 0.01 \\
\hline STE & 0.08 & 0.03 & 0.03 & 0.07 & 0.03 & 0.04 & 0.02 & 0.03 & 0.02 \\
\hline$t$ & 2.93 & -2.28 & 9.36 & -3.83 & 2.32 & 6.54 & -2.89 & -5.68 & 0.34 \\
\hline$Z$ & 2.51 & -1.90 & 6.43 & -3.27 & 1.94 & 5.09 & -2.47 & -4.58 & 0.64 \\
\hline$p$ & 0.0060 & 0.0287 & 0.0000 & 0.0005 & 0.0264 & 0.0000 & 0.0067 & 0.0000 & 0.7387 \\
\hline \multicolumn{10}{|l|}{ Study 3} \\
\hline Coeff & -0.13 & 0.06 & 0.28 & -0.16 & 0.11 & 0.26 & -0.04 & -0.20 & -0.04 \\
\hline STE & 0.07 & 0.03 & 0.04 & 0.06 & 0.04 & 0.04 & 0.03 & 0.05 & 0.04 \\
\hline$t$ & -1.75 & 1.96 & 7.70 & -2.50 & 2.52 & 6.67 & -1.25 & -4.38 & -0.96 \\
\hline Z & -1.35 & 1.58 & 5.87 & -2.13 & 2.15 & 5.22 & -0.78 & -3.75 & -0.41 \\
\hline$p$ & 0.0878 & 0.0571 & 0.0000 & 0.0165 & 0.0159 & 0.0000 & 0.2190 & 0.0001 & 0.3406 \\
\hline \multicolumn{10}{|c|}{ All 3 studies } \\
\hline Coeff & 0.06 & 0.02 & 0.36 & -0.24 & 0.08 & 0.39 & -0.06 & -0.25 & 0.00 \\
\hline STE & 0.05 & 0.03 & 0.03 & 0.04 & 0.02 & 0.04 & 0.02 & 0.03 & 0.02 \\
\hline$t$ & 1.18 & 0.75 & 12.95 & -5.57 & 3.30 & 10.88 & -2.91 & -7.74 & 0.16 \\
\hline$Z$ & 0.70 & 0.11 & 8.13 & -5.01 & 2.99 & 8.13 & -2.60 & -6.62 & 1.14 \\
\hline$p$ & 0.2408 & 0.4543 & 0.0000 & 0.0000 & 0.0014 & 0.0000 & 0.0047 & 0.0000 & 0.8735 \\
\hline
\end{tabular}

The table depicts results from Equation 1 fitted to SV for each of the three studies of dataset 1 separately and for all three studies taken together. The two interactions HC $\times$ HR and $\mathrm{HC} \times \mathrm{TR}$ were the main regressors of interest and were used to calculate a combined regulatory success measure. STE, standard error of the coefficient estimate.

In both GLMs, the subscripts dIFPC and vmPFC refer to the GM volume from those two regions. We assigned anatomical labels based on the MNI coordinates to each set of 91 ROIs, allowing us to identify the dlPFC and vmPFC in each set. Both dlPFC and vmPFC ROIs were present in all 91 training sets. For Equation $3_{\text {all }}$, the subscript $X$ refers to potential additional regressors for any additional ROIs present in that specific training set.

Last, once we had obtained a predicted regulatory success value for each participant from Equation 3 or $3_{\text {all }}$, we quantified the association between predicted and observed regulatory success using Pearson's correlation and a permutation test, which involved estimating the distribution of correlation coefficients by randomly resampling with replacement 10,000 observations for observed and predicted regulatory success.

Predicting out-of-sample regulatory success at the participant and task levels. We also tested whether regulatory success can be predicted in an independent sample of participants (dataset 2, $N=32$ ) performing a different regulation task (i.e., regulation task 2). First, we computed the average GM volume values for each participant in dataset 1 within 5-mm-radius spheres centered around the peak MNI coordinates found within the dIPFC (MNI coordinates $x, y, z(40,40,20)]$ and vmPFC [MNI coordinates $(9,46,-15)]$ when estimating Equation 2 for the full participant sample in dataset 1. Second, we computed the GLM in Equation 3 across all dataset 1 participants to estimate the relationship (i.e., $\beta$ coefficients $\beta_{\mathrm{dlPFC}}$ and $\beta_{\mathrm{vmPFC}}$ ) between vmPFC and dlPFC GM volume and regulatory success. Next, we tested whether regression weights estimated for dataset $1\left(\beta_{\mathrm{dlPFC}}=6.68, \beta_{\mathrm{vmPFC}}=6.92, \beta_{0}=0.0002\right)$ could significantly predict regulatory success on the separate behavioral task used in dataset 2 when combined with the dlPFC and vmPFC GM volumes of those participants. In other words, we used Equation 3 with the intercept set to 0.0002 and GM volume $\beta$ coefficients for dlPFC set to 6.68 , and for vmPFC to 6.92 to make predictions about regulatory success in dataset 2 . Last, we used Pearson's correlation and the same permutation test that was used for testing the results of Equations 3 and 3 all in dataset 1 to quantify the association between the predicted and observed levels of regulatory success $[\mathrm{SV}(\mathrm{NC}-\mathrm{DC})]$ in dataset 2 .

Voxelwise correlations with regulatory success in dataset 2 . To test the relationship between GM volume and regulatory success within dataset 2, we conducted a voxelwise GLM analysis on these data using Equation 4, as follows:

$$
\text { GM volume }=\beta_{0}+\beta_{\text {reg_success }}+\beta_{\text {age }}+\beta_{\text {gender }}+\beta_{\text {global GM }}+\epsilon
$$

This model mirrored the model in Equation 2 except that it omitted study and scanner dummy regressors because all participants in the dataset were part of the same study and thus were scanned with the same MRI scanner. Regulatory success in Equation 4 was defined as the difference in average SV during the NC compared with the DC (i.e., Regulatory Success $\left._{\text {dataset2 }}=S V_{N C}-S V_{D C}\right)$. Once again, voxels in which GM volume was positively associated with regulatory success were identified by the contrast $\left(\beta_{\text {reg_success }}>0\right)$.

\section{Results}

\section{Behavioral results}

Regulatory success when focusing on healthiness during SV computations in dataset 1

We quantified regulatory success in terms of how much participants adjusted the relative weights on healthiness and tastiness in the health focus compared with the natural condition (i.e., the $\mathrm{HC} \times \mathrm{HR}$ and $\mathrm{HC} \times \mathrm{TR}$ interaction terms shown in Fig. $1 b$ ). In line with the previously reported results in the separate original studies, the behavioral GLM described in Equation 1 showed significant interactions between the weightings of the health and taste attributes and the choice conditions in the joint set of 91 participants (Table 2).

These interaction terms capture different forms of regulatory success. Health attributes were significantly more integrated into SV computations in the health focus condition $\left(\beta_{\mathrm{HC} \times \mathrm{HR}}=0.39\right.$; $\left.\mathrm{SEM}_{\mathrm{HC} \times \mathrm{HR}}=0.04 ; t_{(90)}=10.8 ; p<0.001\right)$, indicating that more weight was placed on the healthiness of the foods compared with natural condition. Taste attributes of the foods were significantly less integrated into SV computations in the health focus condition $\left(\beta_{\mathrm{HC} \times \mathrm{TR}}=-0.25 ; \mathrm{SEM}_{\mathrm{HC} \times \mathrm{TR}}=0.03 ; t_{(90)}=-7.74, p<\right.$ 0.001 ), indicating that less weight was placed on the tastiness of the foods compared with the natural condition. The changes in the influence of taste $\left(\beta_{\mathrm{HC} \times \mathrm{TR}}\right)$ and healthiness $\left(\beta_{\mathrm{HC} \times \mathrm{HR}}\right)$ on SV between the $\mathrm{HC}$ and $\mathrm{NC}$ conditions were significantly correlated 
a

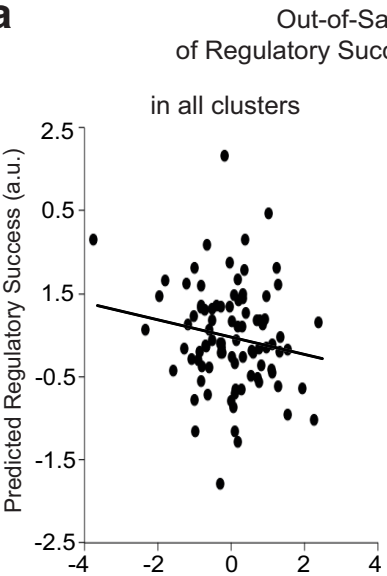

C b

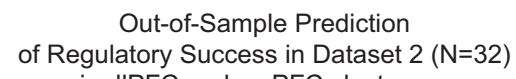

in dIPFC and vmPFC clusters
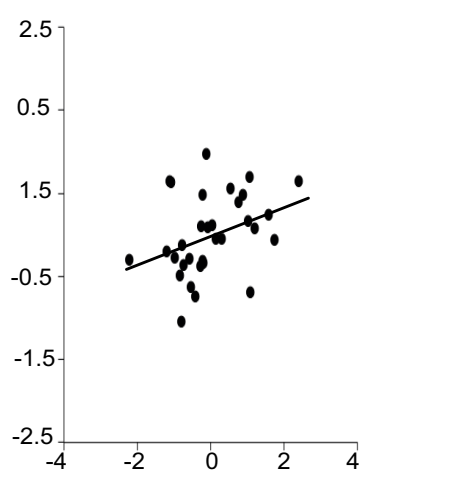

Observed Regulatory Success (z-scored)
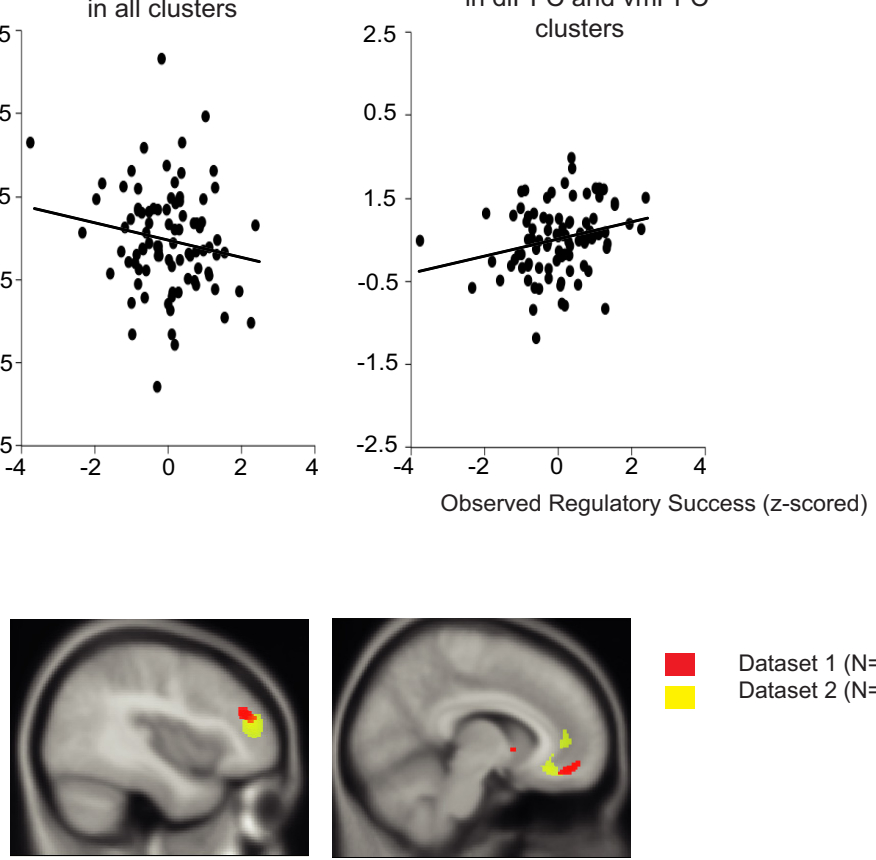

Dataset $1(\mathrm{~N}=91)$ Regulatory success $=\mathrm{HRxHC}-\mathrm{TR} \times \mathrm{HC}$ Dataset $2(\mathrm{~N}=32)$ Regulatory success $=\mathrm{SV}(\mathrm{NC}-\mathrm{DC})$

Figure 2. Neuroanatomical markers of regulatory success in dataset 1 and dataset 2. $\boldsymbol{a}$, Correlation between predicted and observed regulatory success for out-of-sample participants of dataset 1 when considering all clusters (left: Pearson's $r=-0.16, p=0.11$ ) or only vmPFC and dIPFC clusters (right: Pearson's $r=0.25, p=0.02$ ). Dots correspond to participants. $\boldsymbol{b}$, Correlation between predicted and observed regulatory success for out-of-sample participants of dataset 2 when considering only the weights of the vmPFC and dIPFC clusters identified in dataset 1 . $c$, GM volume in the dIPFC and vmPFC significantly correlated with overall regulatory success score (i.e., $\beta_{\mathrm{HC} \times \mathrm{HR}}-\beta_{\mathrm{HC} \times \mathrm{TR}}$ ) of dataset $1(\mathrm{~N}=91$, illustrated in red) and of dataset 2 (i.e., $\mathrm{SV}$ (NC $-\mathrm{DC}$, $N=32$, illustrated in yellow). Significant voxels are displayed for visualization purposes at a whole-brain threshold of $p<0.005$ uncorrected. Statistical parametric maps are superimposed on the average structural brain image of each sample, respectively.

across subjects $(r=0.53, p<0.001)$. Although our primary interest is in the differences between $\mathrm{HC}$ and $\mathrm{NC}$ conditions, we note that there was a significant TC $\times \mathrm{HR}$ interaction $\left(\beta_{\mathrm{TC} \times \mathrm{HR}}=\right.$ $\left.-0.06 ; \mathrm{SEM}_{\mathrm{TC} \times \mathrm{HR}}=0.02 ; t_{(90)}=-2.91, p=0.005\right)$ as well, such that participants were less sensitive to the healthiness of foods in the TC condition. There was no significant TC $\times$ TR interaction.

Regulatory success during SV computation using distancing strategies in dataset 2

Here we briefly restate the behavioral results for participants from dataset 2 . These results are the same as those originally reported in the study by Hutcherson et al. (2012), but are repeated here for the reader's convenience. Participants in dataset 2 showed significantly higher SV values in the indulge condition $\left(\mathrm{IC} ;\right.$ mean $_{\text {Ic zscored }}=0.25$, SEM $\left._{\text {IC zscored }}=0.04\right)$ versus the natural condition $\left(t_{(31)}=6.22, p<0.001 ; 95 \% \mathrm{CI}, 0.17-0.33\right)$. In contrast, they showed significantly lower SV in the distancing condition (mean $\mathrm{SV}_{\mathrm{DC} \text { zscored }}=-0.25, \mathrm{SEM}_{\mathrm{DC} \text { zscored }}=0.04$ ) compared with the natural condition (mean $\mathrm{SV}_{\mathrm{NC} \text { zscored }}=$ $-0.002, \mathrm{SEM}_{\mathrm{NC} \text { zscored }}=0.02 ; t_{(31)}=-6.69 ; 95 \% \mathrm{CI},-0.32$ to $-0.17 ; p<0.001$; Fig. 1d). We used this difference in SV between the distancing and the natural control conditions as the measure of regulatory success for our further analyses in this article.

\section{VBM results}

Anatomical predictors of regulatory success when focusing on healthiness

We were able to significantly predict regulatory success in dataset 1 using GM volume in independently defined dlPFC and vmPFC ROIs and regression weights in a leave-one-subject-out proce- dure. When basing the prediction of regulatory success on information from dIPFC and vmPFC alone, there was a significant positive association between predicted and observed regulatory success (Pearson's $r=0.25, p=0.02 ; 95 \%$ CI due to chance, -0.17 to 0.17 ; Fig. $2 a$ ). In contrast, when using all regions that were correlated with regulatory success in a given training set to predict regulatory success in the test set, there was no significant correlation (Pearson's $r=-0.16, p=0.11 ; 95 \%$ CI due to chance, -0.17 to 0.17 ; Fig. $2 a$ ). The generalization failure of models trained using the GM volume from additional brain regions indicates that these models may be overfitting to the training set. Our results are in line with fMRI studies that have frequently reported the recruitment of the vmPFC and the dlPFC in dietary choices made under both regulatory goals and unregulated conditions (Plassmann et al., 2007, 2010; Hare et al., 2009, 2011; Hutcherson et al., 2012; Harris et al., 2013; van der Laan et al., 2014). In light of these results, we focused on these two regions when attempting to predict regulatory success across choice paradigms using neuroanatomy.

\section{Anatomical markers of regulatory success across regulation} strategies and populations

Next, we tested whether the neuroanatomical correlates of regulatory success identified in regulation task 1 and dataset 1 could be used to make predictions about regulatory success in a separate set of individuals attempting to engage self-regulation in a different type of food choice paradigm (i.e., regulation task 2). In other words, we sought to test how predictive and generalizable the associations between dIPFC and vmPFC GM volume and self-regulation were (Fig. 2b). Thus, we computed $\beta$ weights 
quantifying the association between $\operatorname{dlPFC}\left(\beta_{\mathrm{dIPFC}}=6.68\right)$ and $\operatorname{vmPFC}\left(\beta_{\mathrm{vmPFC}}=6.92\right) \mathrm{GM}$ volumes $\left(\beta_{0}=0.0002\right)$ and the regulatory success measure obtained in dataset 1 (i.e., Eq. 3); and then we used these weights together with the GM volumes measured in these regions for participants in dataset 2 to predict regulatory success in dataset 2 . We found that there was a significant correlation between GM-predicted and observed regulatory success (Fig. $2 b$; Pearson's $r=0.35, p=0.04 ; 95 \%$ CI of correlations due to chance, -0.29 to 0.29 ), indicating that the combination of dIPFC and vmPFC GM volumes can be used to generate significant out-of-sample predictions of regulatory success in different tasks. For robustness, we checked whether the dlPFC and vmPFC separately predicted out-of-sample regulatory success by correlating predicted regulatory success calculated based on the $\beta$ weight and GM volume of each of the two ROIs, respectively. The Pearson correlations between predicted and observed regulatory success were $r=0.28$ and $p=0.11$ for the dlPFC, and $r=0.34$ and $p=0.06$ for the vmPFC. Fisher's $r$-to- $z$ transformation did not detect any significant differences between the two correlations $(z=-0.34 ; p=0.73$, two-tailed).

\section{Whole-brain, voxelwise regression analyses}

We also ran exploratory whole-brain, voxelwise VBM analyses across all participants within both datasets 1 and 2 separately. No regions survived correction for multiple comparisons in either dataset (Tables 3, 4). For illustrative purposes, in Figure $2 c$ we plot voxels in which GM volume correlated with regulatory success in the respective tasks for datasets 1 and 2.

\section{Discussion}

Making healthy food choices is often a challenge in everyday life, and people vary in their ability to choose healthy over tasty foods on the menu, even when they have the explicit goal of eating healthily. This article provides new evidence that regulatory success in healthy eating is related, in part, to individual differences in brain anatomy in both the vmPFC and dIPFC. Importantly, this relationship generalizes across different groups and regulatory strategies. These findings suggest that both brain regions contribute broadly to the regulation of valuation processes in the context of dietary decision-making and its control.

\section{Implications for dietary decision-making and self-control}

Our findings are relevant for current neuroeconomic theories of dietary self-control. Some research in this area suggests that the vmPFC and the dlPFC may represent distinct value systems biased to respond to either immediate hedonistic rewards or delayed, more abstract rewards (McClure et al., 2004; Hutcherson et al., 2012). Other research (Hare et al., 2009) suggests a more cooperative relationship, in which the dlPFC modulates computations in the vmPFC to weight different attributes according to current behavioral goals. Consistent with both theoretical accounts, our results suggest a key role of the vmPFC and the dlPFC for dietary self-control on an anatomical level.

\section{Limitations and open questions}

Our work has several limitations. First, our results do not speak to the question of whether the vmPFC and the dlPFC play differentiable or similar roles in regulatory success. Understanding their specific roles and their interactions is important because of an ongoing debate in the literature regarding different models of self-control: Do they represent two independent sources of value (McClure et al., 2004; Hutcherson et al., 2012), or does the dlPFC play only an indirect role in choice by modulating value signals
Table 3. VBM results in $N=91$ participants (dataset 1): Positive effect of regulatory success

\begin{tabular}{llrrrl}
\hline Region & BA & $x$ & \multicolumn{1}{c}{$y$} & \multicolumn{1}{c}{$z$} & Peakz-score \\
\hline dIPFC & 46 & 40 & 40 & 20 & 3.74 \\
dmPFC & 6 & 15 & 18 & 57 & 3.70 \\
& & 18 & 25 & 60 & 3.20 \\
STG & 22 & 60 & 2 & 0 & 3.22 \\
mPFC & 10 & 4 & 64 & 0 & 3.08 \\
vmPFC & $25 / 11$ & 9 & 46 & -15 & 2.99 \\
\hline
\end{tabular}

This table reports the peak coordinates and $z$-score values for the VBM analysis detailed in Equation 2 across the full sample of 91 participants in dataset 1. All peaks surpassing a voxelwise threshold of $p<0.001$ uncorrected are reported for completeness, but only the dIPFC and vmPFC ROls were used to predict regulatory success across samples. Note that this table is provided as an overview of the results of Equation 2 when fit to dataset 1 and the locations of the dIPFC and vmPFC ROls used to predict regulatory success in dataset 2 , but is not the basis of any statistical inferences in this manuscript. The $x, y$, and $z$ coordinates correspond to the MNI space. STG, Superior temporal gyrus; $\mathrm{mPFC}$, medial prefrontal cortex.

Table 4. VBM results in $N=32$ participants (dataset 2): positive effect of regulatory success

\begin{tabular}{llrrrl}
\hline Region & BA & \multicolumn{1}{l}{$x$} & \multicolumn{1}{l}{$z$} & Peak z-score \\
\hline dIPFC & $46 / 10$ & 42 & 43 & 15 & 4.25 \\
ACC & $32 / 9$ & -12 & 40 & 18 & 4.06 \\
& & 14 & 40 & 2 & 3.37 \\
dACC & & 0 & 18 & 36 & 3.28 \\
PCG & 4 & 55 & -9 & 45 & 4.03 \\
& 6 & 55 & -3 & 12 & 3.42 \\
vmPFC & 25 & 10 & 34 & -15 & 3.70 \\
& 11 & 2 & 26 & -8 & 3.18 \\
AG & 39 & 44 & -56 & 21 & 3.43 \\
\hline
\end{tabular}

This table was obtained by a VBM analysis with a combined regulatory success as a predictor variable of GM volume (Eq. 4) using a whole-brain threshold of $p<0.001$ uncorrected. The $x, y$, and $z$ coordinates correspond to the MNI space. ACC, Anterior cingulate cortex; dACC, dorsal anterior cingulate cortex; PCG, precentral gyrus; AG, angular gyrus.

within the vmPFC (Hare et al., 2009, 2011)? Our results are fully consistent with both models, because dIPFC gray matter volume could either contribute an independent value input to choice processes or provide enhanced capacity to modulate vmPFC value signals. Further work will be needed to tease apart the common and distinct roles the dIPFC and the vmPFC play in regulatory success.

For example, approaches using patients with localized lesions in these brain areas or methods that temporarily inhibit or excite brain activity in these regions will be particularly important. Evidence for a causal role of both regions in human decisionmaking already exists. For example, transcranial magnetic stimulation of the dlPFC produces clear alterations in choice behavior, both in the context of foods (Camus et al., 2009) and in the context of intertemporal decision-making (Figner et al., 2010). Although this latter result is not directly related to healthy decision-making, intertemporal considerations may still play an important role in food choice, which involves trade-offs between the immediately rewarding taste and the longer-term benefits of healthiness in dietary choices. Causal evidence for the role of the vmPFC in dietary and monetary intertemporal choices comes from lesion studies (Sellitto et al., 2010; Camille et al., 2011; Jo et al., 2013; Peters and D'Esposito, 2016). Together then, our results and the results of lesion studies confirm a critical role for both the vmPFC and the dlPFC, but future research investigating their potentially dissociable roles is needed.

Another important question raised by our results is how generalizable the role of individual differences in dIPFC and vmPFC neuroanatomy is beyond the realm of dietary choices. For example, do dIPFC and vmPFC gray matter volumes also predict selfcontrol success for financial decisions when considering saving 
for the future instead of consuming now? There is evidence indicating that individual differences in dIPFC neuroanatomy are related to regulating the intake of addictive substances (Holmes et al., 2016), suggesting a broad and generalizable role for the dlPFC.

\section{Conclusion}

Our findings extend previous work by highlighting the importance of individual differences in the neuroanatomy of the dlPFC and the vmPFC for dietary decision-making and its control. They imply that individual differences in the dIPFC and vmPFC anatomy could be combined with existing assays and measures such as choice, fMRI, or questionnaire data to better estimate an individual's likelihood of success in regulating dietary choices. Our results suggest that regulatory success may result not only from momentary fluctuations in motivation and attention, but also from a more stable variation in neuroanatomy.

Yet the brain and its anatomy are also subject to plasticity in response to new situations, life styles, diseases, and environmental constraints (Merzenich et al., 2013). An exciting avenue going forward will be to explore whether self-control training or biofeedback methods could harness neural plasticity to yield longlasting changes in self-regulatory capacity. Our results suggest that the dIPFC and vmPFC may represent key targets for interventions that alter disadvantageous dietary choices in at-risk populations (e.g., those with obesity or eating disorders).

\section{References}

Camille N, Griffiths CA, Vo K, Fellows LK, Kable JW (2011) Ventromedial frontal lobe damage disrupts value maximization in humans. J Neurosci 31:7527-7532. CrossRef Medline

Camus M, Halelamien N, Plassmann H, Shimojo S, O'Doherty J, Camerer C, Rangel A (2009) Repetitive transcranial magnetic stimulation over the right dorsolateral prefrontal cortex decreases valuations during food choices. Eur J Neurosci 30:1980-1988. CrossRef Medline

Diekhof EK, Gruber O (2010) When desire collides with reason: functional interactions between anteroventral prefrontal cortex and nucleus accumbens underlie the human ability to resist impulsive desires. J Neurosci 30:1488-1493. CrossRef Medline

Diekhof EK, Nerenberg L, Falkai P, Dechent P, Baudewig J, Gruber O (2012) Impulsive personality and the ability to resist immediate reward: an fMRI study examining interindividual differences in the neural mechanisms underlying self-control. Hum Brain Mapp 33:2768-2784. CrossRef Medline

Figner B, Knoch D, Johnson EJ, Krosch AR, Lisanby SH, Fehr E, Weber EU (2010) Lateral prefrontal cortex and self-control in intertemporal choice. Nat Neurosci 13:538-539. CrossRef Medline

Gross JJ (1998) The emerging field of emotion regulation: an integrative review. Rev Gen Psychol 2:271-299. CrossRef

Hare TA, Camerer CF, Rangel A (2009) Self-control in decision-making involves modulation of the vmPFC valuation system. Science 324:646648. CrossRef Medline

Hare TA, Malmaud J, Rangel A (2011) Focusing attention on the health aspects of foods changes value signals in vmPFC and improves dietary choice. J Neurosci 31:11077-11087. CrossRef Medline

Harris A, Hare T, Rangel A (2013) Temporally dissociable mechanisms of self-control: early attentional filtering versus late value modulation. J Neurosci 33:18917-18931. CrossRef Medline

Holmes AJ, Hollinshead MO, Roffman JL, Smoller JW, Buckner RL (2016) Individual differences in cognitive control circuit anatomy link sensation seeking, impulsivity, and substance use. J Neurosci 36:4038-4049. CrossRef Medline

Hutcherson CA, Plassmann H, Gross JJ, Rangel A (2012) Cognitive regula- tion during decision making shifts behavioral control between ventromedial and dorsolateral prefrontal value systems. J Neurosci 32:1354313554. CrossRef Medline

Jo S, Kim KU, Lee D, Jung MW (2013) Effect of orbitofrontal cortex lesions on temporal discounting in rats. Behav Brain Res 245:22-28. CrossRef Medline

Kable JW, Glimcher PW (2007) The neural correlates of subjective value during intertemporal choice. Nat Neurosci 10:1625-1633. CrossRef Medline

Kober H, Kross EF, Mischel W, Hart CL, Ochsner KN (2010) Regulation of craving by cognitive strategies in cigarette smokers. Drug Alcohol Depend 106:52-55. CrossRef Medline

Li N, Ma N, Liu Y, He XS, Sun DL, Fu XM, Zhang X, Han S, Zhang DR (2013) Resting-state functional connectivity predicts impulsivity in economic decision-making. J Neurosci 33:4886-4895. CrossRef Medline

McClure SM, Laibson DI, Loewenstein G, Cohen JD (2004) Separate neural systems value immediate and delayed monetary rewards. Science 306 : 503-507. CrossRef Medline

Merzenich M, Nahum M, Van Vleet TM (2013) Changing brains: applying brain plasticity to advance and recover human ability. In: Progress in Brain Research, Vol 207. Amsterdam, the Netherlands: Elsevier.

Moreno-Lopez L, Contreras-Rodriguez O, Soriano-Mas C, Stamatakis EA, Verdejo-Garcia A (2016) Disrupted functional connectivity in adolescent obesity. Neuroimage Clin 12:262-268. CrossRef Medline

Paschke LM, Dörfel D, Steimke R, Trempler I, Magrabi A, Ludwig VU, Schubert T, Stelzel C, Walter H (2016) Individual differences in self-reported self-control predict successful emotion regulation. Soc Cogn Affect Neurosci 11:1193-1204. CrossRef Medline

Peper JS, Mandl RC, Braams BR, de Water E, Heijboer AC, Koolschijn PC, Crone EA (2013) Delay discounting and frontostriatal fiber tracts: a combined DTI and MTR study on impulsive choices in healthy young adults. Cereb Cortex 23:1695-1702. CrossRef Medline

Peters J, D'Esposito M (2016) Effects of medial orbitofrontal cortex lesions on self-control in intertemporal choice. Curr Biol 26:2625-2628. CrossRef Medline

Pietiläinen KH, Saarni SE, Kaprio J, Rissanen A (2012) Does dieting make you fat? A twin study. Int J Obes (Lond) 36:456-464. CrossRef Medline

Plassmann H, O’Doherty J, Rangel A (2007) Orbitofrontal cortex encodes willingness to pay in everyday economic transactions. J Neurosci 27: 9984-9988. CrossRef Medline

Plassmann H, O’Doherty JP, Rangel A (2010) Appetitive and aversive goal values are encoded in the medial orbitofrontal cortex at the time of decision making. J Neurosci 30:10799-10808. CrossRef Medline

Saarni SE, Rissanen A, Sarna S, Koskenvuo M, Kaprio J (2006) Weight cycling of athletes and subsequent weight gain in middle age. Int J Obes (Lond) 30:1639-1644. CrossRef Medline

Schmidt L, Aron-Wisnewski J, Manoharan N, Chabert M, Leturque A, Clement K, Poitou-Bernert C, Plassmann H (2015) Listen to your gut: How dietary self-regulation is linked to individual differences in hunger hormone levels and delay-discounting for food but not for money. Miami, FL: Society for Neuroeconomics annual meeting.

Sellitto M, Ciaramelli E, di Pellegrino G (2010) Myopic discounting of future rewards after medial orbitofrontal damage in humans. J Neurosci 30:16429-16436. CrossRef Medline

Tangney JP, Baumeister RF, Boone AL (2004) High self-control predicts good adjustment, less pathology, better grades, and interpersonal success. J Pers 72:271-324. CrossRef Medline

Tusche A, Hutcherson CA (2018) Cognitive regulation alters social and dietary choice by changing attribute representations in domain-general and domain-specific brain circuits. eLife 7:e31185. CrossRef

van den Bos W, Rodriguez CA, Schweitzer JB, McClure SM (2014) Connectivity strength of dissociable striatal tracts predict individual differences in temporal discounting. J Neurosci 34:10298-10310. CrossRef Medline

van der Laan LN, de Ridder DT, Viergever MA, Smeets PA (2014) Activation in inhibitory brain regions during food choice correlates with temptation strength and self-regulatory success in weight-concerned women. Front Neurosci 8:308. CrossRef Medline 